\title{
Association of cytomegalovirus and Epstein-Barr virus with cognitive functioning and risk of dementia in the general population : 11-year follow-up study
}

\section{Torniainen-Holm, Minna}

2018-03

Torniainen-Holm , M , Suvisaari , J , Lindgren , M , Härkänen , T , Dickerson , F \& Yolken , R H 2018 , ' Association of cytomegalovirus and Epstein-Barr virus with cognitive functioning and risk of dementia in the general population : 11-year follow-up study ' , Brain, Behavior, and Immunity , vol. 69 , pp. 480-485 . https://doi.org/10.1016/j.bbi.2018.01.006

http://hdl.handle.net/10138/302418

https://doi.org/10.1016/j.bbi.2018.01.006

publishedVersion

Downloaded from Helda, University of Helsinki institutional repository.

This is an electronic reprint of the original article.

This reprint may differ from the original in pagination and typographic detail.

Please cite the original version. 
Full-length Article

\title{
Association of cytomegalovirus and Epstein-Barr virus with cognitive functioning and risk of dementia in the general population: 11-year follow-up study
}

\author{
Minna Torniainen-Holm ${ }^{\mathrm{a}, \mathrm{b}, *}$, Jaana Suvisaari ${ }^{\mathrm{a}}$, Maija Lindgren ${ }^{\mathrm{a}}$, Tommi Härkänen ${ }^{\mathrm{c}}$, Faith Dickerson ${ }^{\mathrm{d}}$, \\ Robert H. Yolken ${ }^{\mathrm{e}}$ \\ ${ }^{a}$ Mental Health Unit, National Institute for Health and Welfare, Helsinki, Finland \\ ${ }^{\mathrm{b}}$ Institute for Molecular Medicine Finland FIMM, University of Helsinki, Helsinki, Finland \\ ${ }^{\mathrm{c}}$ Health Monitoring Unit, National Institute for Health and Welfare, Helsinki, Finland \\ ${ }^{\mathrm{d}}$ Stanley Research Program, Sheppard Pratt Health System, Baltimore, MD, USA \\ e Stanley Division of Developmental Neurovirology, Stanley Neurovirology Laboratory, Johns Hopkins University, School of Medicine, Baltimore, USA
}

\section{A R T I C L E I N F O}

\section{Article history:}

Received 10 October 2017

Received in revised form 2 January 2018

Accepted 14 January 2018

Available online 31 January 2018

\section{Keywords:}

Cytomegalovirus

Epstein-Barr

CMV

EBV

Cognition

Dementia

Neuropsychology

Viral

Prevalence

\begin{abstract}
A B S T R A C T
Background: Earlier studies have documented an association between cytomegalovirus and cognitive impairment, but results have been inconsistent. Few studies have investigated the association of cytomegalovirus and Epstein-Barr virus with cognitive decline longitudinally. Our aim was to examine whether cytomegalovirus and Epstein-Barr virus are associated with cognitive decline in adults.

Method: The study sample is from the Finnish Health 2000 Survey (BRIF8901, $n=7112$ ), which is representative of the Finnish adult population. The sample was followed up after 11 years in the Health 2011 Survey. In addition, persons with dementia were identified from healthcare registers.

Results: In the Finnish population aged 30 and over, the seroprevalence of cytomegalovirus was estimated to be $84 \%$ and the seroprevalence of Epstein-Barr virus 98\%. Seropositivity of the viruses and antibody levels were mostly not associated with cognitive performance. In the middle-aged adult group, cytomegalovirus serointensity was associated with impaired performance in verbal learning. However, the association disappeared when corrected for multiple testing. No interactions between infection and time or between the two infections were significant when corrected for multiple testing. Seropositivity did not predict dementia diagnosis.

Conclusions: The results suggest that adult levels of antibodies to cytomegalovirus and Epstein-Barr virus may not be associated with a significant decline in cognitive function or with dementia at population level.
\end{abstract}

(c) 2018 Published by Elsevier Inc.

\section{Introduction}

Human cytomegalovirus (CMV, also known as human herpesvirus-5, HHV-5) and Epstein-Barr virus (EBV) are among the most common viruses in humans. Both viruses are members of the herpesvirus family and form a lifelong latent infection. CMV and EBV spread mostly through bodily fluids, such as saliva. Primary infections, as well as subsequent virus reactivations, are often asymptomatic or cause only mild symptoms, and therefore most people do not know that they have been infected. However, primary infection or reactivation of the viruses can be potentially

\footnotetext{
* Corresponding author at: Mental Health Unit, National Institute for Health and Welfare, Helsinki, Finland.

E-mail address: minna.torniainen-holm@thl.fi (M. Torniainen-Holm).
}

life-threatening to immunocompromised persons (Emery, 2001; Hodson et al., 2005). In addition, primary infection of CMV during pregnancy may cause cognitive impairment, hearing loss or visual impairment to the unborn baby (Dollard et al., 2007). In Finland, the seroprevalence of CMV was $56 \%$ in pregnant women in South-Western Finland (Alanen et al., 2005), and in another study the seroprevalence in pregnant women was $76 \%$ in a low socioeconomic area and $61 \%$ in a high socioeconomic area of Helsinki (Mustakangas et al., 2000). The seroprevalence of EBV has been estimated to be over 90\% (Cohen, 2000; Puhakka et al., 2016). Infections are slightly more prevalent in women and in persons with low socioeconomic status (Cannon et al., 2010; Dowd et al., 2013).

Two longitudinal studies with approximately 1000 elderly participants found an association between CMV and cognitive decline. 
Barnes et al. noticed an association between CMV seropositivity and cognitive decline and risk of Alzheimer's disease (Barnes et al., 2015). Another study detected an association between CMV immunoglobulin G ( $\operatorname{Ig} G$ ) antibody level (serointensity) and cognitive decline (Aiello et al., 2006). Gow et al. concluded that CMV infection per se may not associate with cognitive ability when controlling for background factors associated with the risk of CMV infection, but having a high level of CMV antibodies associated with a lower level of cognitive ability (Gow et al., 2013). In contrast, Tarter et al. did not find an association between CMV seropositivity and cognitive impairment in a group of 5000 persons over 60 years of age (Tarter et al., 2014).

In non-elderly adults, the association between CMV and cognitive functioning has been studied in few cross-sectional studies with a large sample size. Tarter et al. with almost 5000 middleaged persons, detected an association between CMV seropositivity and cognitive impairment in two out of four cognitive variables (Tarter et al., 2014). Dickerson et al. reported an association between high CMV antibody levels and cognitive functioning in 512 individuals (Dickerson et al., 2014).

The effect of CMV infection on cognitive functioning is expected to be small (Gow et al., 2013; Tarter et al., 2014) and therefore addressing the question requires a large sample size. Previously only a few studies have had adequate sample sizes for investigating the association between CMV and cognitive functioning (Aiello et al., 2006; Barnes et al., 2015; Tarter et al., 2014). In addition, because the risk for CMV infection is associated with many background variables that are also associated with cognitive functioning, the detected association between CMV infection and cognitive functioning could result from not being able to perfectly control for background factors (i.e. residual confounding) (Gow et al., 2013). This issue can be solved by using longitudinal data. Although some longitudinal studies have been published in the elderly (Aiello et al., 2006; Barnes et al., 2015), as far as we know, no longitudinal studies on the effects of CMV infection on cognitive functioning in a non-elderly adult population have been published.

EBV has been less studied than CMV in terms of association with cognitive functioning. Dickerson et al. studied the association between EBV and cognition in two studies: 521 non-elderly persons and 229 persons with schizophrenia, and did not detect any association (Dickerson et al., 2014, 2003). Also in a study with 1000 adolescents, EBV was not associated with cognitive performance (Jonker et al., 2014). However, a meta-analysis with only three small samples found an association between EBV and Alzheimer's disease (Steel and Eslick, 2015).

The purpose of the present study was to examine whether CMV and EBV seropositivity and antibody levels are associated with cognitive decline in adults and the elderly and with dementia diagnosis using a large, representative population sample.

\section{Methods}

\subsection{Participants}

This study is based on the Finnish Health 2000 Survey (BRIF8901), which is a representative, two-stage stratified cluster sample of the Finnish population (Aromaa and Koskinen, 2004). The sample comprised 8028 persons aged 30 years or over living in mainland Finland. Finland was divided into 20 strata based on the five university hospital regions and the 15 largest cities in Finland. From each university hospital region, 16 healthcare districts, including the largest cities, were chosen as clusters. The ultimate sampling units were persons who were selected by systematic random sampling from the healthcare districts. People who were 80 years or older were oversampled using double inclusion probability. The study protocol was approved by the Ethics Committee of the National Public Health Institute and the Hospital District of Helsinki and Uusimaa (HUS) (Heistaro, 2008). Written informed consent was obtained.

Of the total sample, $7112(88.6 \%)$ participated in the health interview and/or health examination. Data were collected between September 2000 and June 2001, including a home interview and a health examination at the local health centre, or for nonrespondents, a condensed interview and health examination performed at home. We excluded persons whose native language was other than Finnish, or was unknown $(n=558)$, from the analyses of cognitive performance. After the exclusion, the study group comprised 6554 persons.

The Health 2000 sample was followed up in 2011 with the Health 2011 Survey. All persons from the Health 2000 sample who were alive, living in Finland and had not refused to participate in any future studies were invited ( $n=5733$ : 5309 persons whose native language was Finnish), and 4620 participated in the Health 2011 Survey (Markkula et al., 2016).

\subsection{Cognitive assessment}

The cognitive variables of the present study can be seen in Table 1. Two tests from the CERAD battery (Moms et al., 1989; Pulliainen et al., 1999) were performed by all participants. For the assessment of verbal fluency, participants were requested to name as many animals as they could within one minute. The number of correct words was recorded.

In the assessment of verbal learning and memory, the participants were shown 10 words one at a time, and were asked to read the words aloud and memorize them. The total number of correctly recalled words was recorded. The words were shown in total three times, each time in a different order. In Health 2000, if the person remembered all the words they were not shown again, and the unshown words were scored as if the person had remembered them. In this study, we used the total number of immediately recalled words after each of the showings as a measure of verbal learning. Short delayed recall after five minutes was categorized for persons who remembered at least $90 \%$, at least $75 \%$ (slightly impaired) and $<75 \%$ (impaired) (Hänninen et al., 2013) of the number of words from the recall in the third showing.

Simple and choice reaction and movement time was recorded by Good Response (Metitur oy, Jyväskylä) (Era et al., 1986). The task was to move the index finger when a light lit up from the waiting switch to the switch that turned the light off as fast as possible. In the simple task, the light lit up in the same place at random intervals. In the choice task, the light lit up in different places in the testing panel. The tasks were repeated 12 times. The response was accepted if the response was given within programmed time limits and was within two standard deviations from the mean of the subject. Mean simple reaction and movement

Table 1

Assessed cognitive variables in two age groups in the baseline and in the 11 year follow-up.

\begin{tabular}{llllll}
\hline & \multicolumn{2}{l}{ Middle-aged adults } & & Elderly & \\
\cline { 2 - 3 } Cognitive variables & Baseline & Follow-up & & Baseline & Follow-up \\
\hline Verbal fluency & $\times$ & $\times$ & $\times$ & $\times$ \\
Verbal learning & $\times$ & $\times$ & $\times$ & $\times$ \\
Verbal short delay recall & $\times$ & $\times$ & $\times$ & $\times$ \\
Simple movement time & $\times$ & & $\times$ & \\
Simple reaction time & $\times$ & & $\times$ & \\
Choice movement time & $\times$ & & $\times$ & \\
Choice reaction time & $\times$ & & $\times$ & \\
Abbreviated Mini-Mental & & & $\times$ & $\times$ \\
$\quad$ State Examination & & & & \\
(MMSE) & & & & \\
\hline
\end{tabular}


times and choice reaction and movement times in seconds were used in the present study. The task was performed only in the baseline assessment.

As a rough estimate for general cognitive functioning an abbreviated version of the Mini-Mental State Examination (MMSE), usually used to assess cognitive decline and screen for dementia (Folstein et al., 1975), was administered during the home interviews for persons who were at least 55 years of age. The subtests included were the assessment of orientation to time (asking for the day, month and year), immediate repeating and delayed recall of three-words, repetition of a phrase, an attention and calculation task of counting down from one hundred by sevens (serial 7s) and copying of a drawing (two intersecting pentagons).

\subsection{Dementia diagnosis}

Persons with dementia in the elderly sample $(n=3065)$ were identified from four registers. From the Hospital Discharge Register and Causes of Death Register we identified persons with the following diagnosis codes: F00, F01, F02, F03, G30 (ICD-10), 3310, 4378A (ICD-9) and 290 (ICD-8/9). Persons who had purchased prescribed, reimbursed medication, or who were entitled to special reimbursement on the basis of Alzheimer's disease or Parkinson's disease diagnosis, were identified from the Prescription Register (ATC class N06D) and Special Reimbursement Register (code 307 by the Social Insurance Institution). Of the elderly, 606 persons had received a dementia diagnosis during the 13-year follow-up by the end of 2013 .

\subsection{CMV and EBV IgG level measurement}

Plasma samples were available from 6250 (88\%) participants. CMV and EBV IgG levels were analysed by solid-phase immunoassay using commercially available assay kits (IBL America) as described by Dickerson et al. (2003). In the present study, we used seropositivity of the infection, which was rated positive if the optimal density value was above or at the infection point in the sample distribution, as well as continuous variables of serointensity.

\subsection{Statistical analysis}

The sampling design was accounted for in the analyses. In addition, post-stratification weights were used to correct for unit nonresponse and oversampling of persons over 80 years of age. Missing values for those who had participated in any part of the study in 2000 ( $n=7112$ ) were imputed by chained equations and random forest imputation using mice package (Van Buuren and Groothuis-Oudshoorn, 2011) in $\mathrm{R}$ version 3.2.2 ( $\mathrm{R}$ Core Team, 2015). Missing values in 2011 were imputed only for those who were alive and living in Finland.

Age and sex standardized seroprevalence in sociodemographic groups using imputed values was calculated as predictive margins using "mimrgns" (Graubard and Korn, 1999) in Intercooled Stata 11.0 (StataCorp, 2009). $\mathrm{R}$ was used for the regression analyses (Lumley, 2004). The analyses of cognitive performance were performed with persons whose native language was Finnish and separately for two age groups: younger (age $<55$ at baseline) and older (age $\geq 55$ at baseline). The association between seropositivity to the viruses and cognitive performance was first analysed. Next, the analyses were repeated with the serointensity of CMV and EBV antibodies. The analyses were controlled for age, sex, years of education, marital status (married or cohabiting vs. others) and urbanicity. To analyse cognitive decline during 11-year follow-up, two-way interactions between time and infection and three-way interaction between the two infections and time were analysed. To ensure that non-linear effects were not missed, we further divided those seropositive for the virus into quintiles of serointensity (CMV ranges were approximately <3.7, 3.7-4.4, 4.5-5.3, 5.3-6.2, $>6.2$ and EBV serointensity ranges $<0.9,0.9-1.2$, $1.2-1.5,1.5-2$ and $\geq 2$ ) and repeated the analyses of cognitive performance with those variables.

The association of the viruses with dementia diagnosis was studied using Cox regression. The analyses were done for persons at least 55 years of age and controlled for age, sex, years of education, marital status, native language and urbanicity.

P-values of $<.05$ were considered statistically significant. Correction for multiple comparisons was performed with false discovery rate using Benjamini-Hochberg procedure (Benjamini and Hochberg, 1995).

\section{Results}

\subsection{Analysis of missingness}

There were no missing values for age, sex, urbanicity or region of residence. Education was missing for $3 \%$ of the participants, marital status for $0.3 \%$ and native language for $0.4 \%$.

Plasma sample for the analysis of the infections was available for 5783 participants whose native language was Finnish (88\%). At baseline, at least a part of cognitive data was available for 6144 (94\%) persons, and 5527 (84\%) persons did not have any missing values in verbal fluency, verbal learning or reaction time tasks. In total, 4324 persons (66\% of the persons at baseline and $81 \%$ of the persons who were alive, had not moved abroad and had not refused further contact) persons participated in the follow-up. Missing information in the variables was predicted by: older age, being a man, having lower education and not being married or cohabiting (Supplementary Table 1). Good performance in verbal fluency was associated with higher probability of performing MMSE and choice reaction time tests. Multiple imputation was used in order to take into account missingness. Registerbased follow-up of dementia diagnoses covered all the participants, and hence there was no missingness.

\subsection{Prevalence of $C M V$ and $E B V$}

The seroprevalence of CMV was estimated to be $84.0 \%$ (95\% CI 83.0-84.9). The seroprevalence of EBV was 98.1\% (95\% CI 97.798.4). Sociodemographic characteristics and seroprevalence of CMV and EBV in sociodemographic groups are shown in Table 2.

\subsection{Association between the viruses and cognitive functioning}

Seropositivity or serointensity of CMV or EBV were mostly not associated with cognitive decline (studied with infection $\times$ time interaction) or cross-sectional cognitive performance in the two age groups after adjusting for age, sex, years of education, marital status (married or cohabiting vs. others) and urbanicity. In the middle-aged adult group, CMV serointensity was associated with impaired performance in verbal learning $(B=-0.12, \mathrm{p}=.04)$ in fourteen models done before correction for multiple comparisons. In the elderly group, CMV serointensity $\times$ time interaction was associated with verbal fluency $(B=0.38, \mathrm{p}=.04)$ and EBV serointen sity $\times$ time interaction $(B=0.63, \mathrm{p}=.05)$ and CMV serointensity $\times$ EBV serointensity $\times$ time interaction $(B=-0.13, \mathrm{p}=.04)$ with verbal learning in sixteen models done before correction for multiple comparisons. However, the associations were non-significant when corrected for multiple testing. We additionally tested interactions with sex and education years, but they were nonsignificant and were removed from the analyses. 
Table 2

Sociodemographic characteristics of the participants and CMV and EBV seroprevalence in sociodemographic groups as predictive margins adjusting for age and sex.

\begin{tabular}{|c|c|c|c|c|c|c|}
\hline & Proportion (\%) or mean & $95 \% \mathrm{CI}$ & CMV (\%) & $95 \% \mathrm{CI}(\%)$ & EBV (\%) & $95 \% \mathrm{CI}(\%)$ \\
\hline Age & 53.0 & $52.6-53.4$ & & & & \\
\hline $30-40$ & 24.0 & $22.9-25.0$ & 73.2 & $70.9-75.6$ & 96.9 & $96.1-97.8$ \\
\hline $41-54$ & 35.2 & $34.1-36.3$ & 83.5 & $81.9-85.1$ & 98.4 & $97.9-98.9$ \\
\hline $55-70$ & 25.4 & $24.4-26.5$ & 90.1 & $88.7-91.5$ & 97.9 & $97.2-98.6$ \\
\hline $71>$ & 15.3 & $14.4-16.2$ & 91.9 & $89.9-94.0$ & 99.3 & $98.1-99.8$ \\
\hline \multicolumn{7}{|l|}{ Sex } \\
\hline Women & 52.5 & $51.3-53.7$ & 86.3 & $85.0-87.7$ & 98.4 & $98.0-98.8$ \\
\hline Men & 47.5 & $46.3-48.7$ & 81.5 & $80.3-82.8$ & 97.7 & $97.1-98.3$ \\
\hline Education years & 11.1 & $10.9-11.2$ & & & & \\
\hline Basic & 40.4 & $38.8-42.0$ & 85.2 & $83.4-87.0$ & 98.2 & $97.6-98.8$ \\
\hline Secondary & 26.5 & $25.3-27.6$ & 85.8 & $84.2-87.5$ & 98.5 & 98.0-99.1 \\
\hline High & 33.1 & $31.7-34.4$ & 81.5 & $80.0-83.1$ & 97.6 & 96.9-98.3 \\
\hline \multicolumn{7}{|l|}{ Marital status } \\
\hline Married or cohabiting & 67.8 & $66.6-68.9$ & 84.8 & $83.7-85.8$ & 98.3 & 97.9-98.7 \\
\hline Single, separated or widowed & 32.2 & $31.1-33.4$ & 81.9 & $80.1-83.8$ & 97.4 & $96.7-98.2$ \\
\hline \multicolumn{7}{|l|}{ Native language } \\
\hline Finnish & 92.9 & 89.9-95.9 & 84.2 & $83.2-85.2$ & 98.1 & $97.7-98.5$ \\
\hline Swedish & 5.3 & $2.5-8.2$ & 77.7 & $74.1-81.2$ & 97.7 & $95.7-99.7$ \\
\hline Other & 1.8 & $1.0-2.6$ & 89.3 & $83.3-95.2$ & 98.9 & $96.8-100$ \\
\hline \multicolumn{7}{|l|}{ Urbanicity } \\
\hline Urban & 61.4 & $55.1-67.6$ & 83.3 & $82.2-84.5$ & 98.2 & $97.8-98.6$ \\
\hline Semi-urban & 14.4 & $8.4-20.4$ & 87.0 & $85.0-88.9$ & 98.2 & $97.3-99.0$ \\
\hline Rural & 24.2 & $18.0-30.5$ & 83.8 & $81.7-85.9$ & 97.7 & $96.7-98.7$ \\
\hline \multicolumn{7}{|l|}{ Region of residence } \\
\hline North & 13.4 & $13.2-13.6$ & 87.7 & $85.8-89.6$ & 98.4 & $97.7-99.1$ \\
\hline East & 17.2 & $16.9-17.5$ & 85.1 & $82.9-87.3$ & 97.6 & $96.6-98.6$ \\
\hline West & 23.0 & $22.5-23.6$ & 85.1 & $82.9-87.3$ & 98.0 & $97.2-98.8$ \\
\hline Southwest & 13.5 & $13.2-13.8$ & 82.8 & $80.4-85.2$ & 98.8 & $98.1-99.4$ \\
\hline South & 32.9 & $32.3-33.5$ & 81.6 & $79.8-83.4$ & 97.9 & $97.1-98.7$ \\
\hline
\end{tabular}

We further checked whether quintiles of CMV and EBV serointensity were associated with cognitive performance in those seropositive for the virus (Fig. 1). EBV quintiles were not associated with cognitive performance. In the middle-aged adults, the third quintile of CMV performed worse than the lowest quintile in verbal fluency $(B=-0.91, \mathrm{p}=.03)$ and in verbal learning $(B=-0.58$, $\mathrm{p}=.007)$. In addition, CMV quintile $\times$ time interaction was significant in verbal learning $(B=0.68, \mathrm{p}=.02)$ between the lowest and the third quintiles, signifying that persons in the third serointensity quintile improved their performance more than persons in the lowest quintile. Also, the second quintile $(B=0.006, \mathrm{p}=.045)$ and the third quintile $(B=0.007, \mathrm{p}=.01)$ had worse performance than the lowest quintile in movement time in the simple task. In the elderly, the highest quintile of CMV performed worse than the lowest quintile in movement time in the choice task $(B=0.03, \mathrm{p}=.02)$. None of the significant results passed the test for multiple comparisons when corrected for fourteen or sixteen models done in the age groups.

Of the elderly group, $17 \%$ received a dementia diagnosis. When using age- and sex-adjusted predictive margins, $17 \%(95 \% \mathrm{CI}$ $15.8 \%-18.5 \%$ ) of persons with and $18 \%$ (95\% CI 12.5\%-23.6\%) of persons without CMV received a dementia diagnosis. With similar estimation, $17 \%$ (95\% CI $16.1 \%-18.5 \%)$ of the persons with EBV and $10.3 \%$ (95\% CI $0.0 \%-21.1 \%$ ) of the persons without EBV received a dementia diagnosis. Seropositivity of CMV ( $\mathrm{HR}=1.32,95 \% \mathrm{CI}$ $0.12-15.0)$ or $\mathrm{EBV}(\mathrm{HR}=2.24,95 \% \mathrm{CI} 0.36-16.4)$, or the interaction between the viruses ( $\mathrm{HR}=0.64,95 \% \mathrm{CI} 0.05-7.65)$, were not associated with a dementia diagnosis. Because of wide confidence intervals, the analysis was confirmed without interaction and the results remained similar (CMV: $\mathrm{HR}=0.85,95 \% \mathrm{CI} 0.57-1.27$; EBV: $\mathrm{HR}=1.74$, 95\% CI 0.51-5.92). The associations were also non-significant using serointensity (CMV: $\mathrm{HR}=0.99,95 \% \mathrm{CI}$ 0.88-1.11; EBV: $1.10,95 \%$ CI $0.83-1.47$; interaction: $\mathrm{HR}=0.99$, 95\% CI 0.93-1.06).

\section{Discussion}

The results of this study suggest that CMV or EBV may not have a significant effect on cognitive functioning or dementia risk in the general population. The two viruses were not associated with cognitive decline over time in middle-aged adults nor the elderly. In the adults, CMV was associated with lower performance in one cognitive variable out of fourteen models done, but the significant association disappeared when corrected for multiple testing.

In the Finnish population aged 30 and over, the seroprevalence of CMV was estimated to be $84 \%$. The prevalence of CMV in people aged $30-40$ was $73 \%$. When considering the fact that the seroprevalence rises with age, our results are close to earlier reported seroprevalences of $56 \%$ and $71 \%$ in pregnant women mostly between 20 and 40 years (Alanen et al., 2005; Mustakangas et al., 2000). The $98 \%$ seroprevalence of the EBV was consistent with results of the earlier study by Puhakka et al. (2016).

The lack of association between CMV and cognitive decline or cognitive performance was unexpected. The results of previous studies have been mixed, but most studies noticed at least some statistically significant association between cognitive functioning and CMV (Aiello et al., 2006; Barnes et al., 2015; Dickerson et al., 2014; Gow et al., 2013; Tarter et al., 2014). The largest study to date, with over 4000 children, 5000 middle-aged adults and 5000 seniors, noticed an association between CMV and cognitive functioning in two out of four variables only in the middle-aged adults, not in the other groups (Tarter et al., 2014). No association between CMV and dementia diagnosis was in contrast with results from Barnes et al. (2015), but in line with other studies with smaller sample sizes (Steel and Eslick, 2015). The results of the present study, together with earlier studies, suggest that if an association between CMV infection and cognitive decline at population level exists, the effect size must be minimal. 

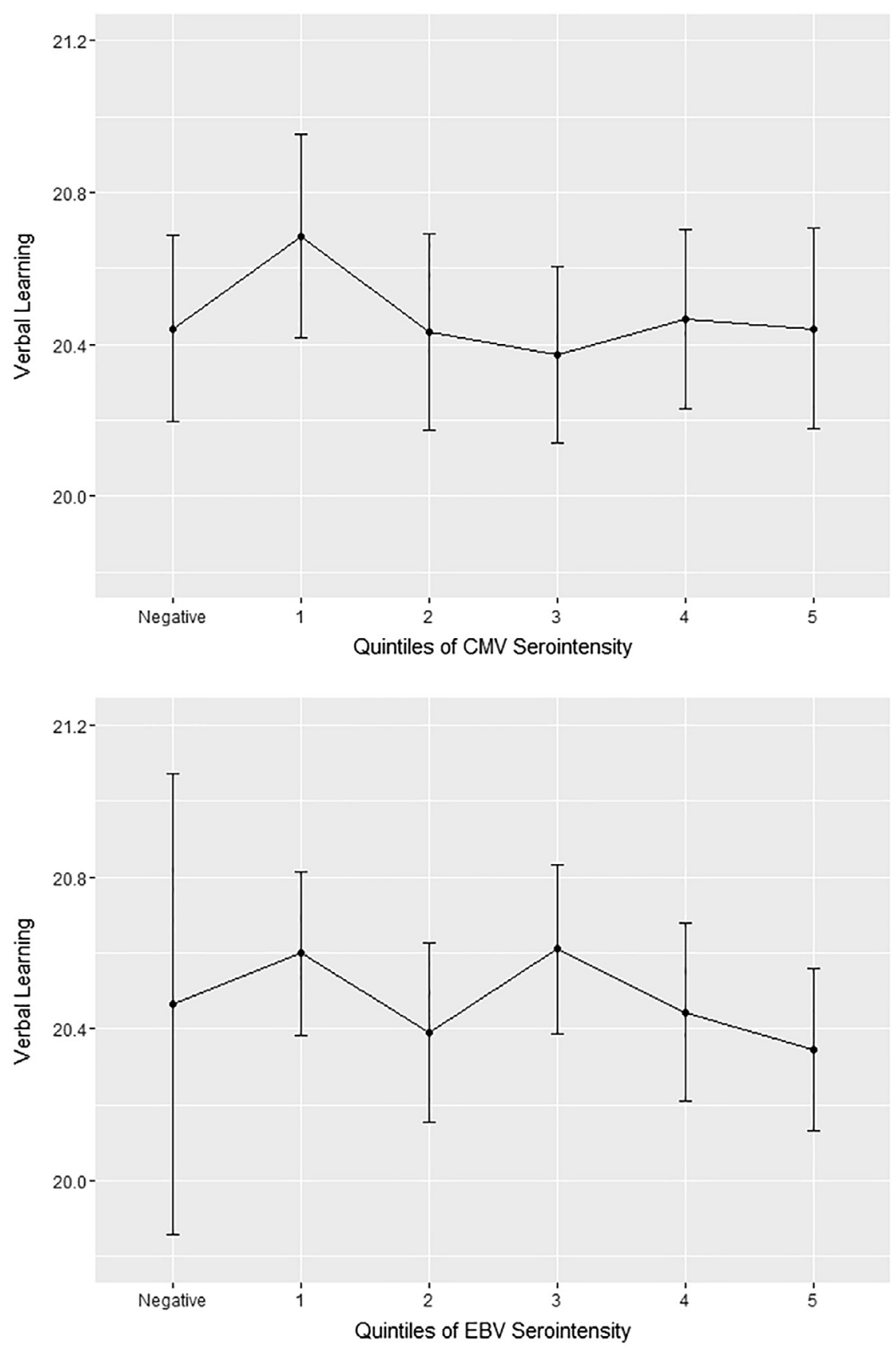

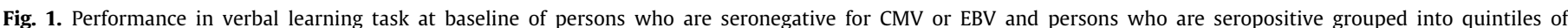

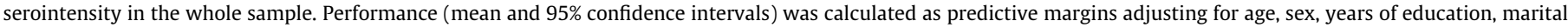
status and urbanicity.

The results of the EBV are accordant with the few earlier studies of no associations with cognitive functioning (Dickerson et al., 2014, 2003; Jonker et al., 2014). We replicated the result with a larger sample size than earlier and with information on change over 11 years. The EBV was not associated with emergent dementia either. The result was in contrast to the results of a metaanalysis of three small samples, which concluded that EBV would associate with the risk of Alzheimer's disease. However, the confidence interval in the present study was wide because almost everyone had EBV, therefore making the interpretation of the results difficult. The analyses with the serointensity of EBV supported the conclusion that EBV would not associate with dementia.

The strengths of the study include a large sample that is representative of the Finnish adult population and a long follow-up time. The participation rate was excellent (89\%) at baseline and lower but still good at the follow-up (71\% of the whole sample). Due to information on non-participants, it was possible to correct for non-response. A weakness of the study is that we did not have a new assessment of the viral antibodies at the follow-up, therefore we were not able to study the effects of seroconversion. In addition, the cognitive test battery used in the present study was short. We excluded persons whose native language was other than Finnish from the analyses of cognitive performance thus possibly limiting the generalizability of the results to people with native language other than Finnish.

\section{Conclusions}

Evidence has been accumulating recently that inflammation could be a cause of neurodegeneration in the elderly (Ransohoff, 
2016). However, the results of the present study suggest that CMV or EBV may not be causative agents of neurodegeneration at population level. Both CMV and EBV are very common infections in the general population and, for most of the people, the viruses cause such mild symptoms that people are unaware of the infection. Possibly, CMV and EBV are associated with cognitive functioning in a subpopulation, such as in persons who are more vulnerable to the effects of virus infection due to other diseases or genetic susceptibility. Because our sample was highly representative of the general population in Finland and because of the large sample size, the result of no association between the two viruses and cognitive decline at population level is plausible.

\section{Acknowledgments}

The study was funded by grant (P50MH094268) from the NIMH (RH Yolken), by the Stanley Medical Research Institute (RH Yolken) and by grant from the Academy of Finland (\#310295, M Torniainen-Holm). The funding sources had no role in the design or implementation of the study, interpretation of the results or preparation of the manuscript. We thank Dr. Glen Ford, VanPelt Biosciences, Montgomery Village, MD, USA, for carrying out the antibody measurements.

\section{Conflict of interest}

None.

\section{Ethical standards}

The authors assert that all procedures contributing to this work comply with the ethical standards of the relevant national and institutional committees on human experimentation and with the Helsinki Declaration of 1975 , as revised in 2008.

\section{Appendix A. Supplementary data}

Supplementary data associated with this article can be found, in the online version, at https://doi.org/10.1016/j.bbi.2018.01.006.

\section{References}

Aiello, A.E., Haan, M.N., Blythe, L., Moore, K., Gonzalez, J.M., Jagust, W., 2006. The influence of latent viral infection on rate of cognitive decline over 4 years. J. Am. Geriatr. Soc. 54, 1046-1054. https://doi.org/10.1111/j.1532-5415.2006.00796.x.

Alanen, A., Kahala, K., Vahlberg, T., Koskela, P., Vainionpää, R., 2005. Seroprevalence, incidence of prenatal infections and reliability of maternal history of varicella zoster virus, cytomegalovirus, herpes simplex virus and parvovirus B19 infection in South-Western Finland. BJOG Int. J. Obstet. Gynaecol. 112, 50-56. https://doi.org/10.1111/j.1471-0528.2004.00320.x.

Aromaa, A., Koskinen, S., 2004. Health and Functional Capacity in Finland. Baseline Results of the Health 2000 Health Examination Survey. Publ. Natl. Public Heal. Inst.. B12/2004.

Barnes, L.L., Capuano, A.W., Aiello, A.E., Turner, A.D., Yolken, R.H., Torrey, E.F., Bennett, D.A., 2015. Cytomegalovirus infection and risk of alzheimer disease in older black and white individuals. J. Infect. Dis. 211, 230-237. https://doi.org/ $10.1093 /$ infdis/jiu437.

Benjamini, Y., Hochberg, Y., 1995. Controlling the false discovery rate: a practical and powerful approach to multiple testing. J. R. Stat. Soc. B. https://doi.org/ $10.2307 / 2346101$.

Cannon, M.J., Schmid, D.S., Hyde, T.B., 2010. Review of cytomegalovirus seroprevalence and demographic characteristics associated with infection Rev. Med. Virol. 20, 202-213. https://doi.org/10.1002/rmv.655.

Cohen, J.I., 2000. Epstein-Barr virus infection. N. Engl. J. Med. 343, 481-492.
Dickerson, F., Stallings, C., Origoni, A., Katsafanas, E., Schweinfurth, L.A.B., Savage, C. L.G., Yolken, R., 2014. Association between Cytomegalovirus antibody levels and cognitive functioning in non-elderly adults. PLoS ONE 9. https://doi.org/ 10.1371 /journal.pone.0095510.

Dickerson, F.B., Boronow, J.J., Stallings, C., Origoni, A.E., Ruslanova, I., Yolken, R.H., 2003. Association of serum antibodies to herpes simplex virus 1 with cognitive deficits in individuals with schizophrenia. Arch. Gen. Psychiatry 60, 466-472. https://doi.org/10.1001/archpsyc.60.5.466.

Dollard, S.C., Grosse, S.D., Ross, D.S., 2007. New estimates of the prevalence of neurological and sensory sequelae and mortality associated with congenital cytomegalovirus infection. Rev. Med. Virol. 17, 355-363. https://doi.org/ $10.1002 /$ rmv.544.

Dowd, J.B., Palermo, T., Brite, J., McDade, T.W., Aiello, A., 2013. Seroprevalence of Epstein-Barr virus infection in U.S. children ages 6-19, 2003-2010. PLoS ONE 8, 1-7. https://doi.org/10.1371/journal.pone.0064921.

Emery, V.C., 2001. Investigation of CMV disease in immunocompromised patients. J. Clin. Pathol. 54, 84-88. https://doi.org/10.1136/jcp.54.2.84.

Era, P., Jokela, J., Heikkinen, E., 1986. Reaction and movement times in men of different ages: a population study. Percept. Mot. Skills 63, 111-130.

Folstein, M.F., Folstein, S.E., McHugh, P.R., 1975. "Mini-mental state": a practical method for grading the cognitive state of patients for the clinician. J. Psychiatr. Res. 12, 189-198.

Gow, A.J., Firth, C.M., Harrison, R., Starr, J.M., Moss, P., Deary, I.J., 2013. Cytomegalovirus infection and cognitive abilities in old age. Neurobiol. Aging 34, 1846-1852. https://doi.org/10.1016/j.neurobiolaging.2013.01.011.

Graubard, B.I., Korn, E.L., 1999. Predictive margins with survey data. Biometrics 55 , 652-659. https://doi.org/10.1111/j.0006-341X.1999.00652.x.

Hänninen, T., Pulliainen, V., Sotaniemi, M., Hokkanen, L., Salo, J., Hietanen, M., Pirttilä, T., Pöyhönen, M., Juva, K., Remes, A., Erkinjuntti, T., 2013. Muistisairauksien tiedonkäsittely-muutosten varhainen toteaminen uudistetulla CERAD-tehtäväsarjalla [Early detection of cognitive changes in memory disorders with CERAD battery]. Duodecim 126, 2013-2022.

Heistaro, S., 2008. Methodology Report. Health 2000 Survey. National Public Health Institute, Helsinki, Finland.

Hodson, E.M., Jones, C.A., Webster, A.C., Strippoli, G.F.M., Barclay, P.G., Kable, K., Vimalachandra, D., Craig, J.C., 2005. Antiviral medications to prevent cytomegalovirus disease and early death in recipients of solid-organ transplants: a systematic review of randomised controlled trials. Lancet 365, 2105-2115. https://doi.org/10.1016/S0140-6736(05)66553-1.

Jonker, I., Klein, H.C., Duivis, H.E., Yolken, R.H., Rosmalen, J.G.M., Schoevers, R.A. 2014. Association between exposure to HSV1 and cognitive functioning in a general population of adolescents. The TRAILS study. PLoS ONE 9, 1-7. https:// doi.org/10.1371/journal.pone.0101549.

Lumley, T., 2004. Analysis of complex survey samples. J. Stat. Softw. 9, 1-19. https:// doi.org/10.18637/jss.v009.i08.

Markkula, N., Härkänen, T., Nieminen, T., Peña, S., Mattila, A.K., Koskinen, S., Saarni, S.I., Suvisaari, J., 2016. Prognosis of depressive disorders in the general population- results from the longitudinal Finnish Health 2011 Study. J. Affect. Disord. 190, 687-696. https://doi.org/10.1016/j.jad.2015.10.043.

Moms, J.C., Heyman, A., Mohs, R.C., Hughes, J.P., van Belle, G., Fillenbaum, G., Mellits, E.D., Clark, C., 1989. The consortium to establish a registry for Alzheimer's Disease (CERAD). Part 1. Clinical and neuropsychological assessment of Alzheimer's disease. Neurology 39, 1159-1165.

Mustakangas, P., Sarna, S., Ammälä, P., Muttilainen, M., Koskela, P., Koskiniemi, M., 2000. Human cytomegalovirus seroprevalence in three socioeconomically different urban areas during the first trimester: a population-based cohort study. Int. J. Epidemiol. 29, 587-591.

Puhakka, L., Sarvikivi, E., Lappalainen, M., Surcel, H.M., Saxen, H., 2016. Decrease in seroprevalence for herpesviruses among pregnant women in Finland: crosssectional study of three time points 1992, 2002 and 2012. Infect. Dis. (Auckl.) 48, 406-410. https://doi.org/10.3109/23744235.2015.1123290.

Pulliainen, V., Hokkanen, L., Salo, J., Hänninen, T., 1999. CERAD, kognitiivinen tehtäväsarja, käsikirja [CERAD, cognitive battery, Manual], Kuopio.

Core Team, R., 2015. A Language and Environment for Statistical Computing. R Foundation for Statistical Computing, Vienna, Austria.

Ransohoff, R.M., 2016. How neuroinflammation contributes to neurodegeneration. Science (80) 353, 777-783. https://doi.org/10.1126/science.aag2590.

StataCorp, 2009. Stata Statistical Software: Release 11. StataCorp LP, College Station, TX.

Steel, A.J., Eslick, G.D., 2015. Herpes viruses increase the risk of Alzheimer's Disease: a meta-analysis. J. Alzheimer's Dis. 47, 351-364. https://doi.org/10.3233/JAD140822.

Tarter, K.D., Simanek, A.M., Dowd, J.B., Aiello, A.E., 2014. Persistent viral pathogens and cognitive impairment across the life course in the third national health and nutrition examination survey. J. Infect. Dis. 209, 837-844. https://doi.org/ 10.1093/infdis/jit616.

Van Buuren, S., Groothuis-Oudshoorn, K., 2011. Multivariate imputation by chained equations. J. Stat. Softw. 45, 1-67. https://doi.org/10.1177/0962280206074463. 